\title{
ToF-SIMS analysis of carbonaceous particles in the sample catcher of the Hayabusa spacecraft
}

\author{
Hiroshi Naraoka ${ }^{1 *}$, Dan Aoki ${ }^{2}$, Kazuhiko Fukushima ${ }^{2}$, Masayuki Uesugi ${ }^{3}$, Motoo Ito ${ }^{4}$, Fumio Kitajima ${ }^{1}$, Hajime Mita ${ }^{5}$, \\ Hikaru Yabuta ${ }^{6}$, Yoshinori Takano ${ }^{7}$, Toru Yada ${ }^{3}$, Yukihiro Ishibashi ${ }^{1}$, Yuzuru Karouji ${ }^{3}$, Takaaki Okada ${ }^{3}$ and \\ Masanao $\mathrm{Abe}^{3}$
}

\begin{abstract}
Three carbonaceous category 3 particles (RA-QD02-0180, RB-QD04-0037-01, and RB-QD04-0047-02) returned in the sample catcher from the Hayabusa spacecraft were analyzed by time of flight-secondary ion mass spectrometry (ToF-SIMS) to establish an analytical procedure for determination of their origins. By the different analytical schemes, the three particles gave distinct elemental and molecular ions, in which the organic carbons commonly appear to be associated with nitrogen, silicon, and/or fluorine. The particles could be debris of silicon rubber and fluorinated compounds and are therefore man-made artifacts rather than natural organic matter.
\end{abstract}

Keywords: Hayabusa spacecraft; Category 3 particle; Carbonaceous material; ToF-SIMS analysis

\section{Correspondence/findings Introduction}

The Hayabusa spacecraft successfully collected surface particles of asteroid Itokawa. The first analysis of the particles by the Hayabusa sample preliminary examination team (HASPET) has revealed a direct link between asteroidal materials and meteorites (e.g., Nakamura et al. 2011; Yurimoto et al. 2011). The particles used for the first analyses were composed mainly of silicate minerals including olivine, pyroxene, and feldspar with minor amounts of sulfide and oxide, which were classified as categories 1 and 2 particles and were confirmed as regolith of Itokawa. As one of the initial analyses, the possible presence of organic matter was explored for categories 1 and 2 particles by Raman and FT-IR spectroscopy (Kitajima et al. 2011) and by amino acid analysis and time of flightsecondary ion mass spectrometry (ToF-SIMS) analysis of the solvent extracts. However, no indigenous carbonaceous materials or organic compounds were identified in these particles (Naraoka et al. 2012). Even though investigations of categories 1 and 2 particles have been made over the world, there is no report of carbonaceous materials.

\footnotetext{
* Correspondence: naraoka@geo.kyushu-u.ac.jp

'Department of Earth and Planetary Sciences, Kyushu University, 6-10-1

Hakozaki, Higashi-ku, Fukuoka 812-8581, Japan

Full list of author information is available at the end of the article
}

Category 3 particles are defined as having predominantly carbon signatures based on their chemical composition using field emission scanning electron microscope (FE-SEM) with energy dispersion spectrometer (EDS) at Extraterrestrial Sample Curation Center (ESCuC) in JAXA. In contrast to the category 4 particles defined as man-made artifacts (aluminum flakes, quartz glass, and stainless steel) from the sample catcher or micromanipulator, the origins of category 3 particles are not clear. Currently, 58 particles have been classified into category 3 from the 459 particles categorized at ESCuC/JAXA (Uesugi et al. 2014). Apart from the dominant carbon (C), the category 3 particles often show small nitrogen $(\mathrm{N})$ and oxygen $(\mathrm{O})$ peaks with trace amounts of fluorine and sulfur peaks by SEM/EDS.

Time of flight-secondary ion mass spectrometry (ToFSIMS) has been applied to chemical analysis of solid surfaces with a high spatial resolution (approximately submicrometer) using primary ion beams of gallium $(\mathrm{Ga})$, gold $(\mathrm{Au})$, bismuth $(\mathrm{Bi})$ as well as $\mathrm{C}_{60}, \mathrm{Au}_{3}$, or $\mathrm{Bi}_{3}$ clusters for primary ionization (e.g., Mahoney 2009). Both positive and negative secondary ions of metals and organic fragments from the sample surface can be analyzed by ToF-SIMS with a mass resolution of approximately $10,000(\mathrm{~m} / \Delta \mathrm{m}$ at $m / z$ approximately 400 ). In this study, three category 3 particles were analyzed by ToF-SIMS using $\mathrm{Au}^{+}$ion beams to establish a method in the analytical sequence

\section{它}

(c) 2015 Naraoka et al.; licensee Springer. This is an Open Access article distributed under the terms of the Creative Commons Attribution License (http://creativecommons.org/licenses/by/4.0), which permits unrestricted use, distribution, and reproduction in any medium, provided the original work is properly credited. 
of Itokawa grains and to clarify the chemical composition related to their origins.

\section{Samples}

The category 3 particles are generally $c a$. 20 to $100 \mu \mathrm{m}$ in size (major axis) with blocky or fibrous shape. The blocky samples sometimes show irregular features including a horn-like texture possibly produced by tearing. They often contain distinct small grains of silicate, stainless steel, and aluminum on their surfaces by the SEM/EDS analyses (Uesugi et al. 2014). SEM analyses have been performed at lower electron energy (approximately $10 \mathrm{keV}$ ) under approximately $60 \mathrm{~Pa} \mathrm{~N}_{2}$ atmosphere using a dry (i.e., oilfree) pumping system to avoid the damage and contamination of particle samples (Yada et al. 2014). After the SEM observation, each particle was fixed onto a gold $(\mathrm{Au})$ or indium (In) plate (0.3 $\mathrm{mm}$ in thickness) by pressing with 5-mm-thick sapphire glass under $\mathrm{N}_{2}$ atmosphere at ESCuC (Uesugi et al. 2014). Three category 3 particles (RB-QD04-0047-02, RB-QD04-0037-01, and RA-QD020180) were allocated for ToF-SIMS analysis. These were the typical particles of the blocky-shaped samples, which were classified as type I (38 of a total of 52 category 3 particles; Uesugi et al. 2014). The samples are described in the following paragraphs in their analytical order.

RB-QD04-0047-02 is a blocky particle (approximately $28 \mu \mathrm{m}$ in size) composed of only $\mathrm{C}$ and $\mathrm{O}$. This particle is apparently very hard with no deformation noticeable during the sample processing, whereas other particles were deformed or crushed during the handling (Uesugi et al. 2014). The particle was pressed onto a Au plate. After the FT-IR measurement at ESCuC/JAXA, NanoSIMS analysis was performed at Kochi Institute for Core Sample Research of JAMSTEC to measure stable isotope ratios of hydrogen $(\delta \mathrm{D})$, carbon $\left(\delta^{13} \mathrm{C}\right)$, and nitrogen $\left(\delta^{15} \mathrm{~N}\right)$ in order to discriminate the particle origins: extraterrestrial material vs. terrestrial contamination (Ito et al. 2014). The sample was rastered with the focused $\mathrm{Cs}^{+}$beam after coating of the surface with $\mathrm{Au}$ (10 to $20 \mathrm{~nm}$ ) for mitigation of electrical charging. The detailed procedure of NanoSIMS analysis was given elsewhere (Ito et al. 2014).

RB-QD04-0037-01 is a blocky particle (approximately 48 $\mu \mathrm{m})$ showing major $\mathrm{C}, \mathrm{N}$, and $\mathrm{O}$ peaks by SEM/EDS with tiny olivine grains (approximately $3 \mu \mathrm{m}$ ) on the surface of the particle. The particle was crushed into two fragments during mounting onto the In plate. Only one fragment was subjected to ToF-SIMS analysis prior to NanoSIMS analysis to prevent damaging the sample surface. Even though both RB-QD04-0047-02 and RB-QD04-0037-01 samples were preserved in a $\mathrm{N}_{2}$-purged container during transportation, the sample loading to ToF-SIMS needed about 30 min of exposure to normal atmospheric conditions.

RA-QD02-0180 is also a blocky particle (approximately $55 \mu \mathrm{m}$ ) characterized by $\mathrm{K}, \mathrm{Na}$, and $\mathrm{Cl}$ peaks in addition to $\mathrm{C}, \mathrm{N}$, and $\mathrm{O}$ by SEM/EDS. The elemental signature suggested the existence of salts, which may be deliquescent under atmospheric conditions. Therefore, this sample was handled with a special care under a $\mathrm{N}_{2}$ atmosphere for transportation and loading for the ToF-SIMS analysis. The $\mathrm{O}_{2-}$ and $\mathrm{H}_{2} \mathrm{O}$-free analytical system called the cryoToF-SIMS/SEM system has been reported elsewhere (Kuroda et al. 2013; Masumi et al. 2014).

\section{Analytical methods}

The ToF-SIMS measurement was performed using a TRIFT III spectrometer (ULVAC-PHI, Inc., Chigasaki, Kanagawa, Japan) at Nagoya University. Positive and negative ion spectra were obtained using a $22-\mathrm{keV} \mathrm{Au}^{+}$gold primary ion at a current of $1.2 \mathrm{nA}$, with a pulse width of $1.4 \mathrm{~ns}$ (bunched for spectral analysis, spectrum mode) or $8.0 \mathrm{~ns}$ (not bunched for image analysis, image mode). The angle between the primary $\mathrm{Au}^{+}$ion beam and the sample surface was $40^{\circ}$. The measured surface areas were $30 \times 30$ to $80 \times 80 \mu \mathrm{m}$, and approximately 2 million total ion counts were obtained in acquisition time of about $5 \mathrm{~min}$. A low-energy pulsed electron ion gun $(28.0 \mathrm{eV})$ was used for surface charge compensation.

\section{Results and discussion \\ Alteration and contamination during sample preparation}

To characterize and categorize the Hayabusa particles for allocation, every particle has been analyzed initially by the SEM/EDS at ESCuC (Yada et al. 2014; Uesugi et al. 2014). It has been known that the surface of the organic materials is damaged by electron beams and that amorphous carbon films build up where the electron beams impinge by way of various carbon contaminants including oils, gasket materials, and other residual gases in the common SEM system (e.g., Knox 1976; Bret et al. 2005). To minimize the damage and organic contamination, the SEM analysis of ESCuC was performed at lower electron energy $(10 \mathrm{keV})$ under approximately 60 $\mathrm{Pa} \mathrm{N}_{2}$ atmosphere using the dry (i.e., oil-free) pumping system. Because the surface of organic polymers can be damaged by electron energies as low as a few tens of electron volts (Gilmore and Seath 2002), the organic surface could be altered and contaminated by the SEM analyses as well as during the sample preparation. Nevertheless, the distinctive ion distributions have been observed for each particle as follows:

\section{RB-QD04-0047-02}

RB-QD04-0047-02 gave a strong positive ion signal of $\mathrm{m} / z 132.91$ (1.6 million counts of total approximately 2 million counts) due to $\mathrm{Cs}^{+}$(Figure 1), because this sample was subjected previously to NanoSIMS analysis in which the focused Cs beam was rastered over the sample as a primary ion (Ito et al. 2014). In addition to the 


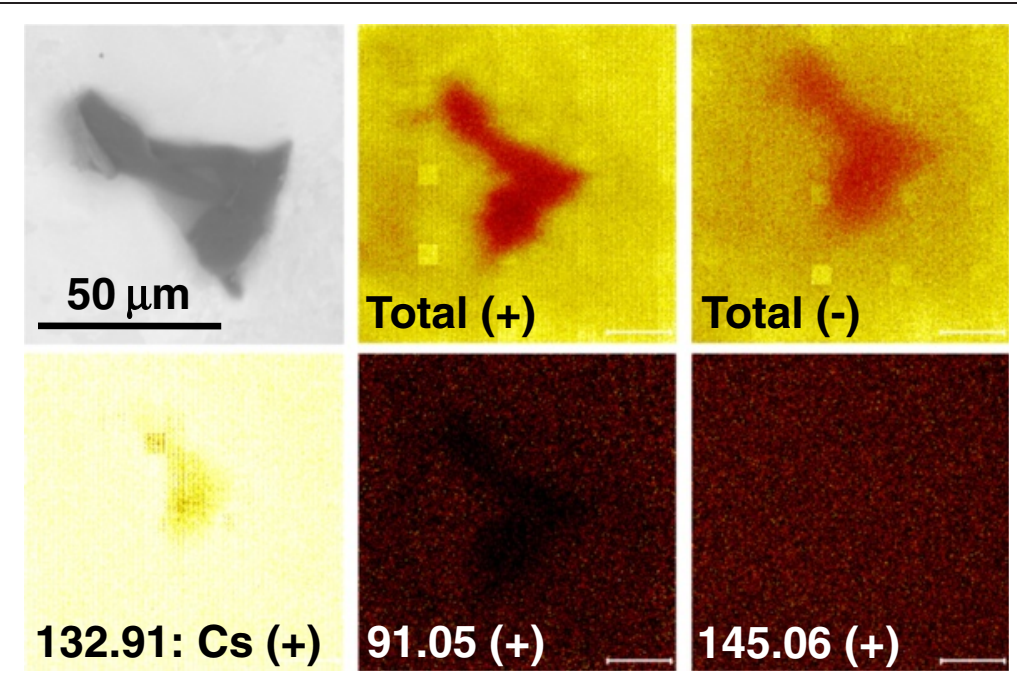

Figure 1 Images of secondary ion distributions of RB-QD04-0047-02 by ToF-SIMS before pre-sputtering. Images of secondary positive (+) and negative (-) ion distributions of RB-QD04-0047-02 by ToF-SIMS (before pre-sputtering) with its backscattered electron image by SEM. The brighter color corresponds to higher concentrations of secondary total ion and specific ion $(\mathrm{m} / \mathrm{z})$. Note that intense ions were emitted more from the background rather than the particle itself. Scale bars of ToF-SIMS analysis are $10 \mu \mathrm{m}$.

strong Cs signal, positive ions of $\mathrm{m} / \mathrm{z} 265.81,329.87$, 462.78, 659.74, and 856.71 were prominent, which were assigned to the species $\mathrm{Cs}_{2}^{+}, \mathrm{AuCs}^{+}, \mathrm{AuCs}_{2}^{+}, \mathrm{Au}_{2} \mathrm{Cs}_{2}^{++}$, and $\mathrm{Au}_{3} \mathrm{Cs}_{2}^{+}$, respectively. Besides these metal ions, metal oxide ions such as $\mathrm{AuCsO}^{+}\left(\mathrm{m} / z\right.$ 345.87), $\mathrm{AuCs}_{2} \mathrm{O}_{2}^{+}(\mathrm{m} / z$ 494.77), and $\mathrm{AuCsO}_{2} \mathrm{H}^{+}(\mathrm{m} / z$ 362.87) were also observed. For negative ions, $\mathrm{Au}^{-}$ion $(m / z$ 196.97) was the strongest followed by $\mathrm{AuO}_{2}^{-}\left(\mathrm{m} / z\right.$ 228.96) and $\mathrm{O}_{2}^{-}(\mathrm{m} / \mathrm{z}$ 31.99). Other negative ions of $\mathrm{Au}$ and its oxides including $\mathrm{Au}_{2}^{-}(m / z$ 393.93 $)$ and $\mathrm{Au}_{3}^{-}\left(m / z\right.$ 590.90), $\mathrm{AuO}_{2}^{-}$ ( $m / z$ 260.95), $\mathrm{Au}_{2} \mathrm{O}_{2}^{-}$( $m / z$ 425.92), $\mathrm{Au}_{2} \mathrm{O}_{4}^{-}(m / z$ 457.91), $\mathrm{Au}_{3} \mathrm{O}_{2}^{-}\left(m / z\right.$ 622.89), and $\mathrm{Au}_{3} \mathrm{O}_{4}^{-}(m / z$ 654.88) were also identified. These $\mathrm{Au}$-related ions are attributable to the Au coating for the NanoSIMS analysis and/or Au plate for the sample mounting. Although several weak positive ions such as $\mathrm{m} / \mathrm{z} 91.05$ and 145.06 were observed (Figure 1), these ions were emitted primarily from the surrounds of the particle rather than the particle itself $(\mathrm{m} / \mathrm{z}$ $91.05)$ or equally from the rastered region $(m / z$ 145.06).

Because the surface of RB-QD04-0047-02 was severely damaged by the Cs ion beam of the NanoSIMS analysis, the sample surface was sputtered using a $\mathrm{Au}$ ion beam for $3 \mathrm{~s}$ three times to remove the altered surface. After the pre-sputtering, negative ions gave distinct ion responses (Figure 2), which may be indigenous to the particle. The region of interest (ROI) was defined to reconstruct the mass spectra of the particle relative to those of the background as shown in Additional file 1: Figure S1A-G. A strong negative ion at $m / z 26.00$ was assigned as $\mathrm{CN}^{-}$ followed by $m / z \quad 12.00\left(C^{-}\right)$and $m / z 24.00\left(C_{2}^{-}\right)$. Further polycarbon ions including $\mathrm{C}_{3}^{-}(\mathrm{m} / \mathrm{z} 36.00), \mathrm{C}_{4}^{-}(\mathrm{m} / z \mathrm{z} .00)$, $\mathrm{C}_{5}^{-}(\mathrm{m} / z 60.00)$, and $\mathrm{C}_{6}^{-}(\mathrm{m} / z$ 72.00) were also detected with probable $\mathrm{C}_{3} \mathrm{~N}^{-}(\mathrm{m} / z$ 50.00) based on the similar spatial distribution as $\mathrm{CN}^{-}$, suggesting that this particle was a lump of $\mathrm{C}$ with minor $\mathrm{N}$. Interestingly, halogens such as $\mathrm{F}^{-}(m / z 19.00)$ and $\mathrm{Cl}^{-}(m / z 34.97$ and 36.97) were also apparent (Additional file 1: Figure S1B).

In contrast, the positive ion became stronger in $\mathrm{m} / \mathrm{z}$ $132.88\left(\mathrm{Cs}^{+}\right)$after pre-sputtering, in which more than $90 \%$ of the total ions were due to $\mathrm{Cs}^{+}$. Except for slight increases of $m / z 12.00\left(\mathrm{C}^{+}\right)$and $26.98\left(\mathrm{Si}^{+}\right)$that were observed using ROI (Additional file 1: Figure S1A), there is no difference in the ion signal between the particle and the blank. The identification of $\mathrm{Si}^{+}$(observed at $\mathrm{m} / \mathrm{z}$ 27.977 for a true mass of 27.9764) was correctly made to discriminate from $\mathrm{CO}^{+}(\mathrm{m} / z 27.995$ for 27.9944) and $\mathrm{C}_{2} \mathrm{H}_{4}^{+}(m / z 28.031$ for 28.0308$)$ as shown in Additional file 1: Figure S1C.

\section{RB-QD04-0037-01}

A strong positive ion signal at $\mathrm{m} / z 114.88$ with minor $\mathrm{m} / z 112.89$ was observed due to $\mathrm{In}^{+}$emitted from the surrounds of the particle. Only faint signals of $\mathrm{m} / z 12.00$ $\left(\mathrm{C}^{+}\right)$and $27.98\left(\mathrm{Si}^{+}\right)$were recognized in mass imaging (Figure 3). On the other hand, negative ions gave some spectra of $m / z 12.00,13.01,19.00$, and 26.00, which were assigned to $\mathrm{C}^{-}, \mathrm{CH}^{-}, \mathrm{F}^{-}$, and $\mathrm{CN}^{-}$, respectively (Figure 3 ). However, these ion distributions were not clearly distinguished as originating from the particle because the mass spectra of $m / z 12.00,13.01$, and 26.00 were emitted from the surrounds of the particle too.

Since it was fairly effective to sputter the surface of particle before analysis, the sample was sputtered with Au ion twice for 1-s and 2-s durations. After the sputtering, some 

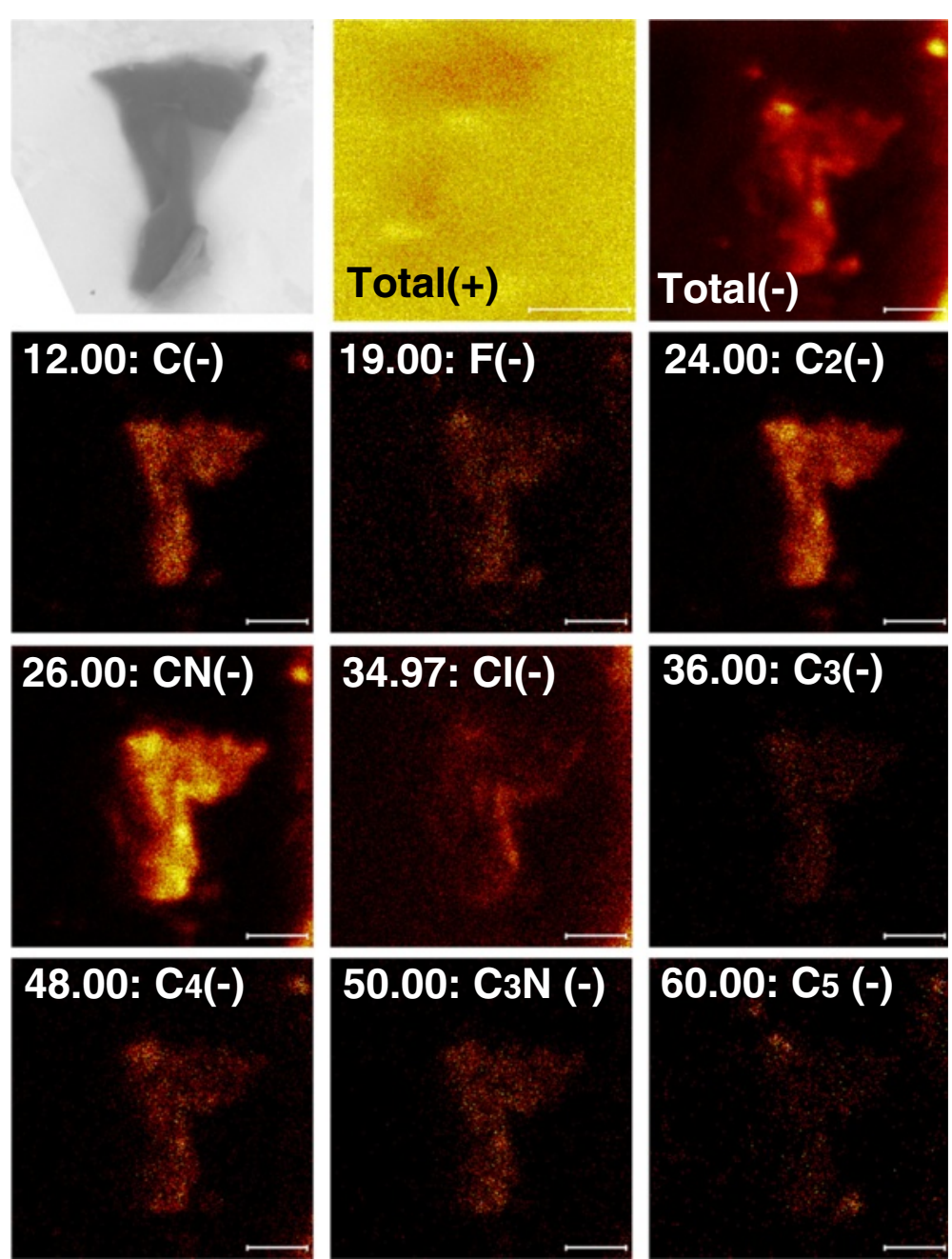

Figure 2 Images of secondary ion distributions of RB-QD04-0047-02 by ToF-SIMS after pre-sputtering. Images of secondary positive (+) and negative (-) ion distributions of RB-QD04-0047-02 by ToF-SIMS (after pre-sputtering) with its backscattered electron image by SEM. The brighter color corresponds to higher concentrations of secondary total ion and specific ion $(\mathrm{m} / \mathrm{z})$. Scale bars of ToF-SIMS analysis are $10 \mu \mathrm{m}$.

mass spectra became obvious as shown in Figure 4 and Additional file 1: Figure S1D, E. In the positive ions, $\mathrm{Si}^{+}$ $(\mathrm{m} / z$ 27.976; Additional file 1: Figure S1C) was characterized from the entire surface of the particle. Note the presence of small area (approximately $3 \mu \mathrm{m}$ in major axis) with relatively strong intensities of $m / z 23.99\left(\mathrm{Mg}^{+}\right)$and 55.94 $\left({ }^{56} \mathrm{Fe}^{+}\right)$with faint $m / z 56.94\left({ }^{57} \mathrm{Fe}^{+}\right)$, which could be derived from a silicate mineral such as olivine. The small area of $\mathrm{Mg}^{+}$and $\mathrm{Fe}^{+}$was consistent with the presence of olivine grains on the surface of the particle by SEM observation. However, the $\mathrm{Si}$ distribution was not coincident with the $\mathrm{Mg}^{+}$and $\mathrm{Fe}^{+}$distributions, suggesting that $\mathrm{Si}$ was not attributable to silicates but possibly from other $\mathrm{Si}$ bearing organic compounds. Negative ions also became stronger with sputtering, in which $\mathrm{CN}^{-}(\mathrm{m} / z$ 26.00) is dominant followed by $\mathrm{C}^{-}(m / z 12.00), \mathrm{C}_{2}^{-}(\mathrm{m} / z 24.00)$, and $\mathrm{CH}^{-}\left(m / z\right.$ 13.01) with weak intensities of $\mathrm{C}_{3}^{-}(m / z 36.00)$ and $\mathrm{C}_{4}^{-}(\mathrm{m} / \mathrm{z} 48.00)$. In contrast, the signal of $\mathrm{F}^{-}(\mathrm{m} / \mathrm{z}$ 19.00) became weaker after sputtering, and the $\mathrm{Cl}^{-}(\mathrm{m} / z$ 34.97) ion had a similar distribution as $\mathrm{F}^{-}$. Fluorine may have existed on the surface of particle and is possibly a contaminant.

\section{RA-QD02-0180}

This particle was characterized by strong positive ions of $m / z 22.99\left(\mathrm{Na}^{+}\right)$and $38.97\left(\mathrm{~K}^{+}\right)$, which are consistent with the observation by SEM/EDS. Although these alkaline metals were widely distributed on the particle, positive ions of $m / z 12.00\left(\mathrm{C}^{+}\right)$and $27.98\left(\mathrm{Si}^{+}\right)$were also emitted from the particle (Figure 5 and Additional file 1: Figure S1F). In the negative ions, fluorine $(\mathrm{m} / z$ 19.00) was distinguished followed by $\mathrm{Cl}^{-}\left(\mathrm{m} / z\right.$ 34.97) and $\mathrm{CN}^{-}$ $(\mathrm{m} / z 26.00)$, of which the distributions were similar to those of $\mathrm{Na}^{+}$and $\mathrm{K}^{+}$. However, the ion distribution was 

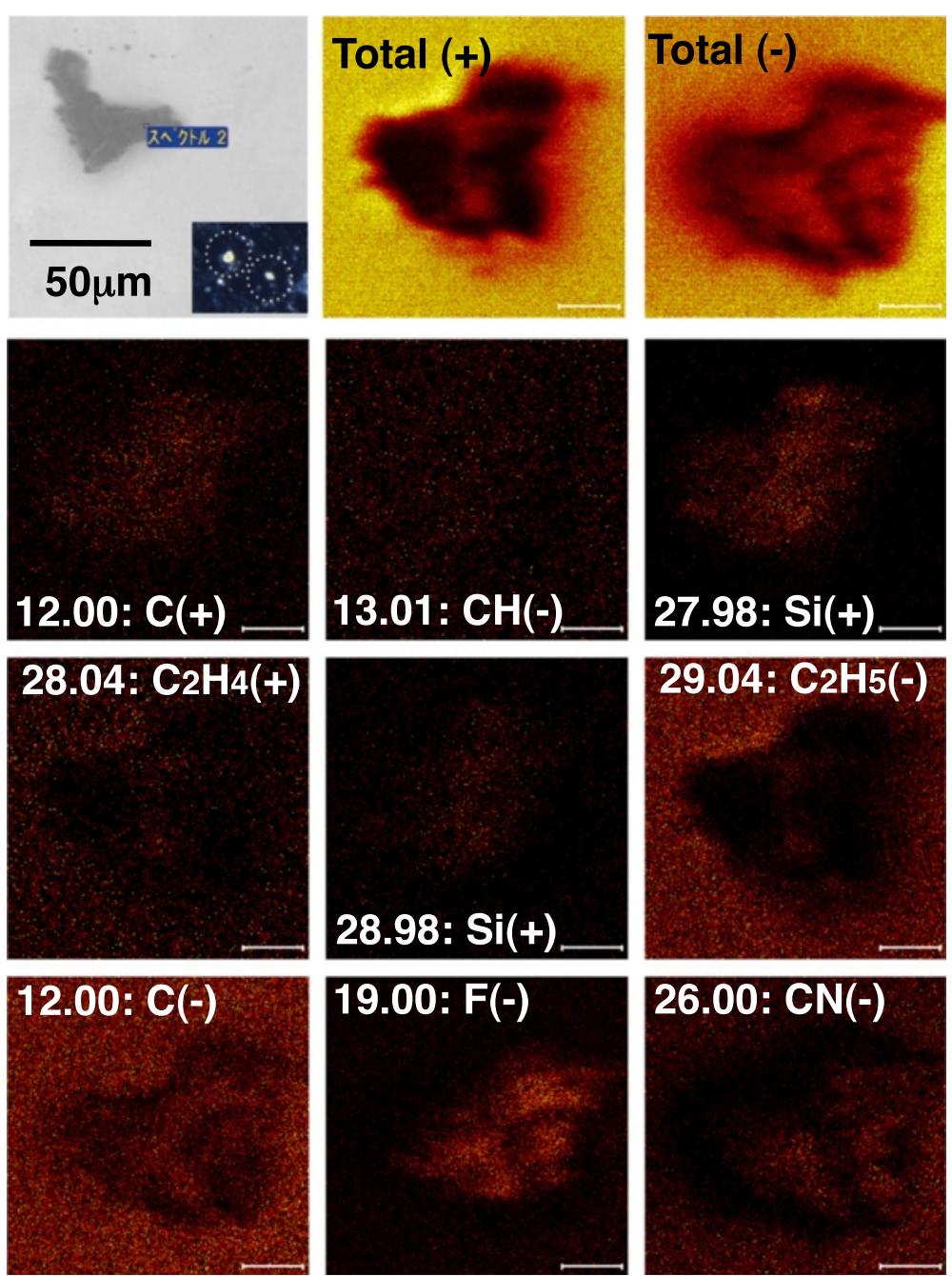

Figure 3 Images of secondary ion distributions of RB-QD04-0037-01 by ToF-SIMS before pre-sputtering. Images of secondary positive (+) and negative (-) ion distributions of RB-QD04-0037-01 by ToF-SIMS (before pre-sputtering) with its backscattered electron image by SEM. The original particle was crushed into two large pieces during mounting onto In plate, shown as two circles in the SEM image. The left particle was analyzed in this study. The brighter color corresponds to higher concentrations of secondary total ion and specific ion ( $\mathrm{m} / \mathrm{z}$ ). Scale bars of ToF-SIMS analysis are $10 \mu \mathrm{m}$.

slightly different between $\mathrm{F}$ and $\mathrm{Cl}$. While the $\mathrm{Cl}^{-}$distribution was generally related to the distributions of $\mathrm{Na}^{+}$and $\mathrm{K}^{+}$, the $\mathrm{F}^{-}$distribution also matched closely with the $\mathrm{C}^{+}$distribution and weakly with the $\mathrm{Si}^{+}$and $\mathrm{CH}^{-}$distribution. Therefore, fluorine may be composed of organo-silicon compounds in addition to making salts with $\mathrm{Na}^{+}$and $\mathrm{K}^{+}$. Using the ROI in Additional file 1: Figure S1G, $m / z 65.995$ was also apparent, possibly assigned to $\mathrm{CF}_{3}^{-}$(68.9958). Although this sample was not subjected to pre-sputtering by $\mathrm{Au}$ ion for the subsequent NanoSIMS analysis, the C distribution was similar to the Si and F distributions.

\section{Analytical sequence}

Various analytical methods are preferred to characterize the properties of category 3 particles (Uesugi et al.
2014). An initial analysis of the Hayabusa particles has been carried out by SEM/EDS on a copper $(\mathrm{Cu})$ holder under low pressure of $\mathrm{N}_{2}$, where the particle can be handled by micromanipulation. In contrast, ToF-SIMS and NanoSIMS analyses of the microparticles require fixing of particle on the metal (e.g., Au or In) or the use of an organic adhesive. Although the organic adhesive was not used, to avoid organic contamination in the present study, the Au or In plate gave a strong background of $\mathrm{m} / z 197$ and 115, respectively, resulting in weak emission of other ions from the particle. The reconstruction of mass spectra using ROI is effective in discriminating the ion peaks of the particle relative to those of the background. In addition, several ions assigned as organic fragments were emitted from the metal (Figure 1). The 

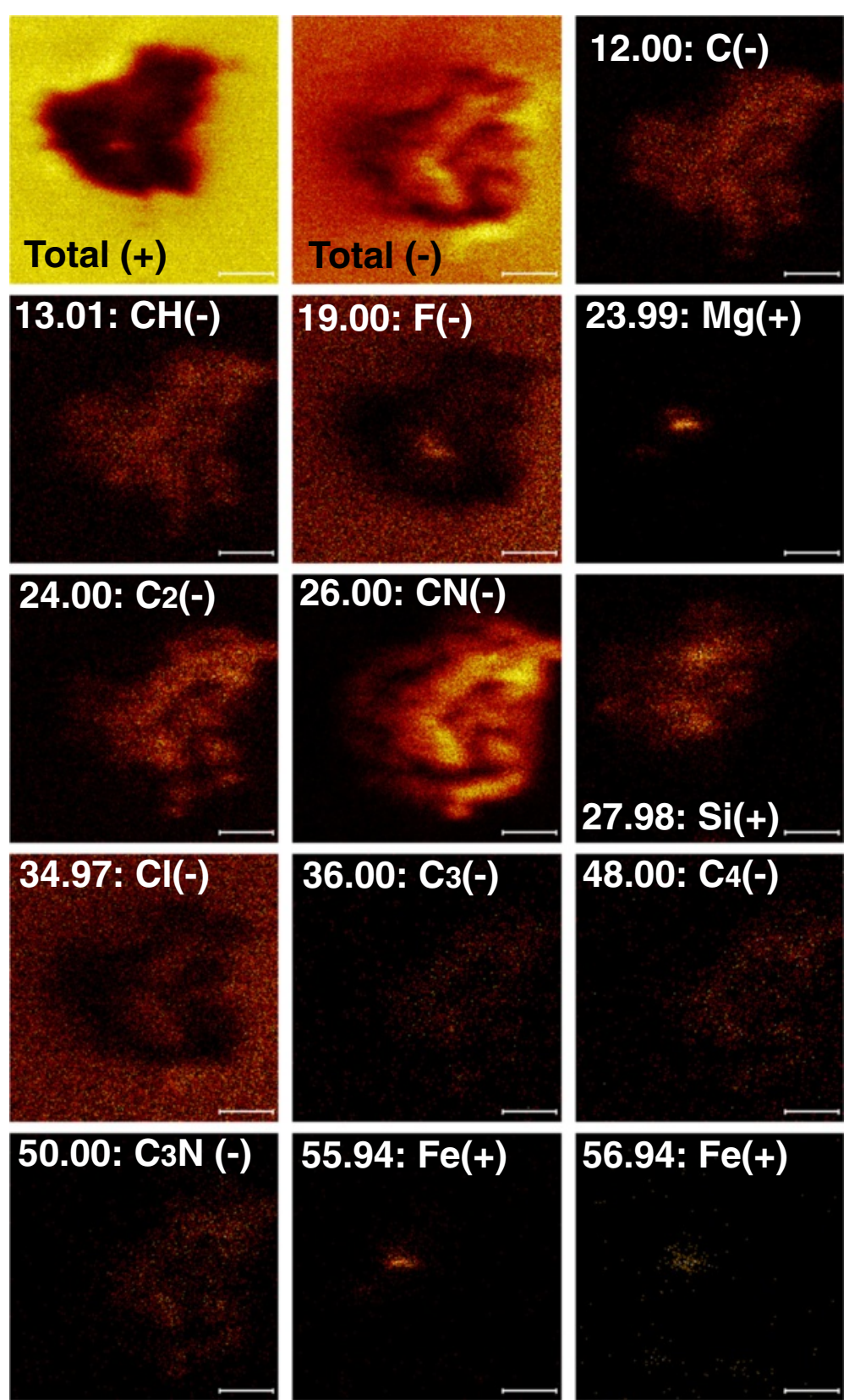

Figure 4 Images of secondary positive (+) and negative (-) ion distributions of RB-QD04-0037-01 by ToF-SIMS (after pre-sputtering). The brighter color corresponds to higher concentrations of secondary total ion and specific ion ( $\mathrm{m} / \mathrm{z})$. Scale bars of ToF-SIMS analysis are $10 \mu \mathrm{m}$.

pre-sputtering by $\mathrm{Au}^{+}$beam could be effective in removing contamination from the sample surface. Furthermore, it is recommended that the ToF-SIMS analysis will be performed prior to the SEM analysis, because the organic surface is damaged by electron beams during the SEM analysis.

The NanoSIMS analysis prior to ToF-SIMS analysis had a great influence on ion distributions shown by ToF-SIMS, because the Cs ion was too strong to observe indigenous positive ions from the sample. Even if the pre-sputtering was performed prior to analysis, the intensity of $\mathrm{Cs}^{+}$got stronger resulting in no effective measurement of positive secondary ions. ToF-SIMS analysis is highly recommended before NanoSIMS analysis, even though stable isotopic ratios of $\mathrm{C}, \mathrm{N}$, and $\mathrm{H}$ may have been slightly enriched in heavy isotopes after the ToF-SIMS analysis (Ito et al. 2014). The $\delta^{13} \mathrm{C}, \delta^{15} \mathrm{~N}$, and $\delta$ D values of RB-QD04-0047-02 before the ToF-SIMS analysis were $+3 \%( \pm 3)$ (relative to $\mathrm{PDB}),-4 \%( \pm 2)$ (relative to Air), and $+103 \%$ (relative to $\mathrm{SMOW}$ ), respectively, 


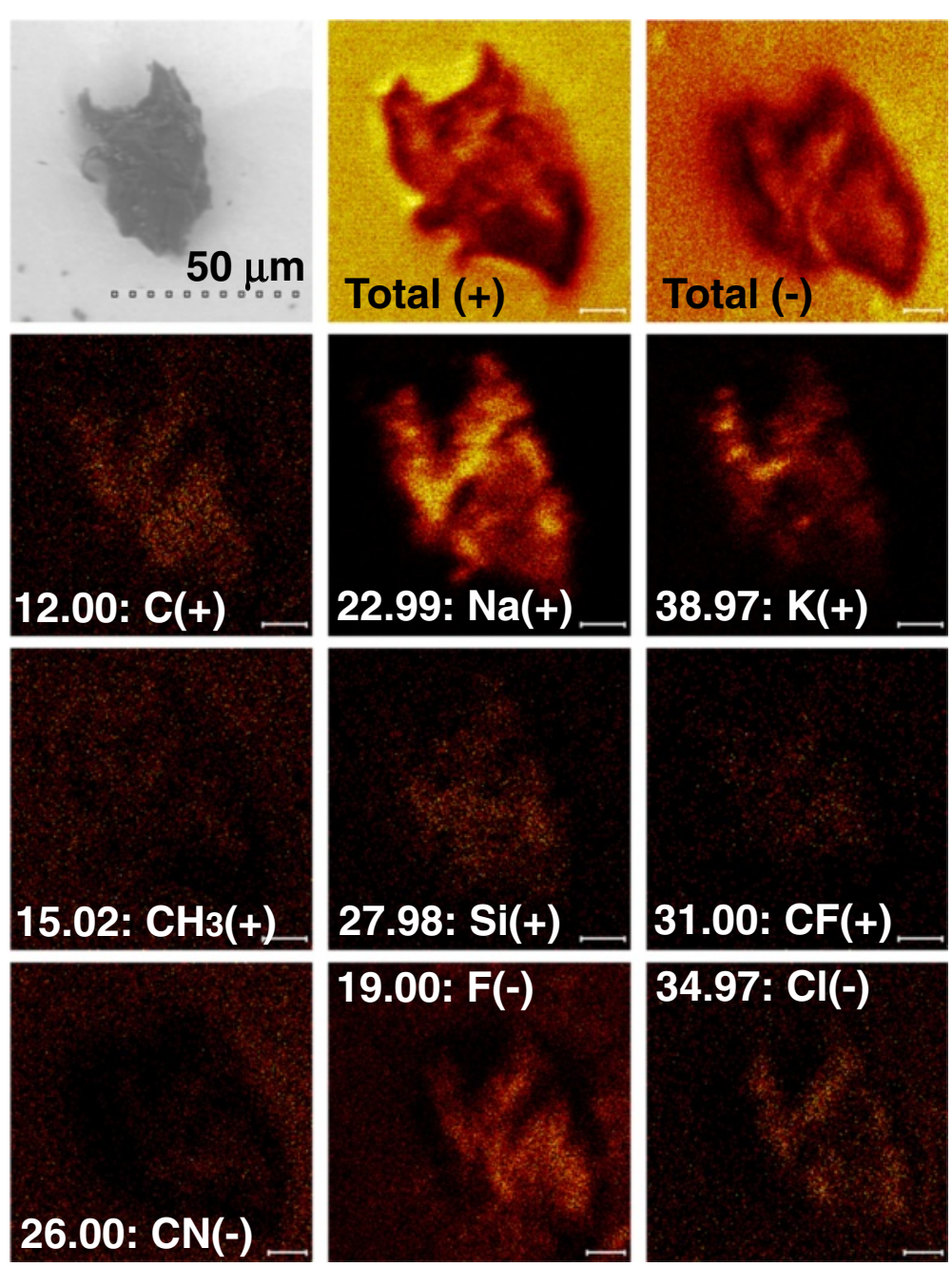

Figure 5 Images of secondary ion distributions of RA-QD02-0180 by ToF-SIMS. Images of secondary positive (+) and negative (-) ion distributions of RA-QD02-0180 by ToF-SIMS with its backscattered electron image by SEM. The brighter color corresponds to higher concentrations of secondary total ion and specific ion $(\mathrm{m} / \mathrm{z})$. Scale bars of ToF-SIMS analysis are $10 \mu \mathrm{m}$.

whereas those after the ToF-SIMS analysis were $+14 \%$ $( \pm 6),+6 \%( \pm 2)$, and $+177 \%$, respectively (Ito et al. 2014$)$.

Furthermore, because a primary beam sputters the surface of sample during SIMS analysis, IR spectroscopic measurements should be performed before ToF-SIMS analysis. Some absorption lines of IR spectra were lost after the SIMS analyses (Uesugi et al. 2014).

\section{Characteristics of category 3 particles by ToF-SIMS for their origins}

Carbon was detected in all samples of this study by SEM/EDS, and the ToF-SIMS analysis also confirmed carbon distribution in all samples. Based on carbon distribution, each particle is a homogenous organic material except for small olivine grains of RB-QD04-0037-01 and $\mathrm{NaCl}$ of RA-QD02-0180 on the surface. Nitrogen was commonly associated with $\mathrm{C}$, and the distributions of fluorine and silicon are similar to those of $\mathrm{C}$ in $\mathrm{RB}$ QD04-0047-02 and RB-QD04-0037-01, respectively. On the other hand, extraterrestrial organic materials including insoluble organic matter (IOM) of meteorites are highly heterogeneous in elemental and isotope distributions (e.g., Busemann et al. 2006; Zega et al. 2010; Ito et al. 2014). Even though the cell materials in terrestrial biological samples also show chemical heterogeneities (e.g., membrane, nucleus, organelle, etc.), artificial organic polymers have usually homogeneous chemical compositions on a micrometer scale. In this study, however, chemical and isotopic heterogeneities have not been observed in any particle. In addition, it is important to note that the Si- and F-bearing organic matter usually has not been found in natural environments except for man-made chemicals. The three particles of this study are probably artifacts in origin, even though each has 
different histories such as cosmic-ray exposure duration, chemical alteration, and mechanical deformation. The man-made chemical origin of the particles is also consistent with the stable isotopic compositions of $\mathrm{C}, \mathrm{H}$, and $\mathrm{N}$ for terrestrial materials (Ito et al. 2014).

The specific source(s) of the original chemicals is (are) uncertain in the present study. A possible source is the outer cover of the sampler horn of the Hayabusa spacecraft, which was made of polyarylate (Vectran ${ }^{\circ}$; Kuraray Co. Ltd., Chiyoda, Tokyo, Japan), although polyarylate usually does not contain Si and F. Silicon rubber is used as the seal materials of clean rooms, and fluoro-rubber (Viton ${ }^{\circ}$ DuPont, Wilmington, DE, USA) is used for the gloves of the clean chambers at ESCuC (Uesugi et al. 2014). However, they usually do not contain N. Alteration of the particle surface by the SEM analysis also may be significant. Further investigations are needed to identify the specific source(s).

\section{Conclusions}

Three carbonaceous category 3 particles (RA-QD02-0180, RB-QD04-0037-01, and RB-QD04-0047-02) collected from the sample catcher of the Hayabusa spacecraft were analyzed by ToF-SIMS. Multiple analytical techniques can be carried out on the same sample, in which case it is preferable that the ToF-SIMS analysis should be performed before the NanoSIMS analysis to avoid severe contamination from the NanoSIMS primary Cs ion beam. It is also recommended that the ToF-SIMS analysis be carried out before the SEM/EDS analysis to avoid damage and contamination of the surface by electron beam interactions. The pre-sputtering of the sample surface by the primary $\mathrm{Au}^{+}$ion beam of ToF-SIMS may be effective in removing contamination from the sample surface. Although the three particles showed distinct elemental and molecular ion distributions, the homogenous organic carbon distribution appears to be associated with nitrogen, silicon, and fluorine, which are different to the elemental distributions of $\mathrm{C}$-rich extraterrestrial materials. The particles could be debris of silicon or fluorinated organic compounds and as such are man-made artifacts rather than natural organic matter.

\section{Additional file}

Additional file 1: Figure S1A. Positive mass spectra of RB-QD04-0047-02 using ROI after pre-sputtering. a) Total MS, b) ROI of particle, and c) other ROI. Figure S1B. Negative mass spectra of RB-QD04-0047-02 using ROI after pre-sputtering. a) Total MS, b) ROI of particle, and c) other ROI. Figure S1C. High-resolution mass spectra to discriminate $\mathrm{Si}, \mathrm{CO}$, and $\mathrm{CH}$. a) Total MS and b) ROI of particle of RB-QD04-0047-02 after pre-sputtering; c) total MS before pre-sputtering, d) total MS after pre-sputtering, e) ROI of particle after pre-sputtering of RB-QD04-0037-01; f) total MS and g) ROl of particle of RA-QD02-0180. Figure S1D. Positive mass spectra of RB-QD04-0037-01 using ROI after pre-sputtering. a) Total MS, b) ROI of particle, and c) other ROI. Figure S1E. Negative mass spectra of RB-QD04-0037-01 using ROI after pre-sputtering. a) Total MS, b) ROI of particle, and c) other ROI. Figure S1F. Positive mass spectra of RA-QD02-0180 using ROl. a) Total MS, b) ROl of particle, and c) other ROI. Figure S1G. Negative mass spectra of RA-QD02-0180 using ROI. a) Total MS, b) ROI of particle, and c) other ROI.

\section{Competing interests}

The authors declare that they have no competing interests.

\section{Authors' contributions}

HN, DA, and KF performed the ToF-SIMS analysis, data interpretation, and manuscript preparation. MU, TY, YI, and YK carried out the sample preparation. MI, FK, HM, HY, YT, TO, and MA contributed the design of the research and interpretation. All authors approved the final manuscript.

\section{Acknowledgements}

We are grateful to two anonymous reviewers and the handling editor, Prof. Trevor Ireland of Australian National University, for critical and constructive comments, which helped to revise an earlier version of this manuscript.

\section{Author details}

${ }^{1}$ Department of Earth and Planetary Sciences, Kyushu University, 6-10-1 Hakozaki, Higashi-ku, Fukuoka 812-8581, Japan. ${ }^{2}$ Graduate School of Bioagricultural Sciences, Nagoya University, Furo-cho, Chikusa-ku, Nagoya, Aichi 464-8601, Japan. ${ }^{3}$ Institute of Space and Astronautical Science, Japan Aerospace Exploration Agency (ISAS/JAXA), 3-1-1 Yoshinodai, Chuo-ku, Sagamihara, Kanagawa 252-5210, Japan. ${ }^{4}$ Kochi Institute for Core Sample Research, Japan Agency for Marine-Earth Science and Technology (JAMSTEC), B200 Monobe, Nankoku, Kochi 783-8502, Japan. ${ }^{5}$ Department of Life, Environment and Materials Science, Fukuoka Institute of Technology, 3-30-1 Wajiro-higashi, Higashi-ku, Fukuoka 811-0295, Japan. ${ }^{6}$ Department of Earth and Space Sciences, Osaka University, 1-1 Machikaneyama-cho, Toyonaka, Osaka 560-0043, Japan. ${ }^{7}$ Department of Biogeochemistry, JAMSTEC, 2-15 Natsushima, Yokosuka, Kanagawa 237-0061, Japan.

Received: 30 April 2014 Accepted: 20 February 2015

Published online: 10 May 2015

\section{References}

Bret T, Mauron S, Utke I, Hoffmann P (2005) Characterization of focused electron beam induced carbon deposits from organic precursors. Microelectron Eng 78-79:300-306

Busemann H, Young AF, Alexander CM, O'D HP, Mukhopadhyay S, Nittler LR (2006) Interstellar chemistry recorded in organic matter from primitive meteorites. Science 312:727-730

Gilmore IS, Seath MP (2002) Electron flood gun damage in the analysis of polymers and organics in time-of-flight SIMS. Appl Surf Sci 187:89-100

Ito M, Uesugi M, Naraoka H, Yabuta H, Kitajima F, Mita H, Takano Y, Karouji Y, Yada T, Ishibashi Y, Okada T, Abe M (2014) H, C and N isotopic compositions of HAYABUSA category 3 organic samples. Earth Planet Space 66:91

Kitajima F, Kotsugi M, Ohkochi T, Naraoka H, Ishibashi Y, Abe M, Fujimura A, Okazaki R, Yada T, Nakamura T, Noguchi T, Nagao K, Tsuchiyama A, Mukai T, Sandford S A, Okada T, Shirai K, Ueno M, Yoshikawa M, Kawaguchi J (2011) A micro-spectroscopic approach to the carbonaceous matter in the particles recovered by the Hayabusa mission (abstract \#1855). 42nd Lunar and Planetary Science Conference, Lunar and Planetary Institute, Houston.

Knox WA (1976) Contamination formed around a very narrow electron beam. Ultramicroscopy 1:175-180

Kuroda K, Fujiwara T, Imai T, Takama R, Saito K, Matsushita Y, Fukushima K (2013) The cryo-TOF-SIMS/SEM system for the analysis of the chemical distribution in freeze-fixed Cryptomeria japonica wood. Surf Interface Anal 45:215-219

Mahoney C M (2009) Cluster secondary ion mass spectrometry of polymers and related materials. Mass Spectrom Rev. doi 10.1002/mas.20233

Masumi T, Matsushita Y, Aoki D, Takama R, Saito K, Kuroda K, Fukushima K (2014) Adsorption behavior of poly(dimethyl-diallylammonium chloride) on pulp fiber studied by cryo-time-of-flight secondary ion mass spectrometry and cryo-scanning electron microscopy. Appl Surf Sci 289:155-159

Nakamura T, Noguchi T, Tanaka M, Zolensky ME, Kimura M, Tsuchiyama A, Nakato A, Ogami T, Ishida H, Uesugi M, Yada T, Shirai S, Fujimura A, Okazaki R, Sandford SA, Ishibashi Y, Abe M, Okada T, Ueno M, Mukai T, Yoshikawa M, 
Kawaguchi J (2011) Itokawa dust particles: a direct link between S-type asteroids and ordinary chondrites. Science 333:1113-1116

Naraoka H, Mita H, Hamase K, Mita M, Yabuta H, Saito K, Fukushima K, Kitajima F, Sandford SA, Nakamura T, Noguchi T, Okazaki R, Nagao K, Ebihara M,

Yurimoto H, Tsuchiyama A, Abe M, Shirai K, Ueno M, Yada T, Ishibashi Y, Okada T, Fujimura A, Mukai T, Yoshikawa M, Kawaguchi J (2012) Preliminary organic compound analysis of microparticles returned from Asteroid 25143 Itokawa by the Hayabusa mission. Geophys J Roy Astron Soc 46:61-72

Uesugi M, Naraoka H, Ito M, Yabuta H, Kitajima F, Takano Y, Mita H, Ohnishi I, Kebukawa Y, Yada T, Karouji Y, Ishibashi Y, Okada T, Abe M (2014) Sequential analysis of carbonaceous materials in Hayabusa-returned sample for the determination of their origin. Earth Planet Space 66:102

Yada T, Fujimura A, Abe M, Nakamura T, Noguchi T, Okazaki R, Nagao K, Ishibashi Y, Shirai K, Zolensky ME, Sandford S, Okada T, Uesugi M, Karouji Y, Ogawa M, Yakame S, Ueno M, Mukai T, Yoshikawa M, Kawaguchi J (2014) Hayabusa return sample curation in the planetary material sample curation facility of JAXA. Meteorite Planetary Sci 49:135-153

Yurimoto H, Abe K, Abe M, Ebihara M, Fujimura A, Hashiguchi M, Hashizume K, Ireland TR, Itoh S, Katayama J, Kato C, Kawaguchi J, Kawasaki N, Kitajima F, Kobayashi S, Meike T, Mukai T, Nagao K, Nakamura T, Naraoka H, Noguchi T, Okazaki R, Park C, Sakamoto N, Seto Y, Takei M, Tsuchiyama A, Uesugi M, Wakaki S, Yada T et al (2011) Oxygen isotopic compositions of asteroidal materials returned from Itokawa by the Hayabusa mission. Science 333:1116-1119

Zega TJ, Alexander CM, O'D BH, Nittler LR, Hoppe P, Stroud RM, Young AF (2010) Mineral associations and character of isotopically anomalous organic material in the Tagish Lake carbonaceous chondrite. Geochim Cosmochim Acta 74:5966-5983

\section{Submit your manuscript to a SpringerOpen ${ }^{\circ}$ journal and benefit from:}

- Convenient online submission

- Rigorous peer review

- Immediate publication on acceptance

Open access: articles freely available online

- High visibility within the field

- Retaining the copyright to your article

Submit your next manuscript at $>$ springeropen.com 